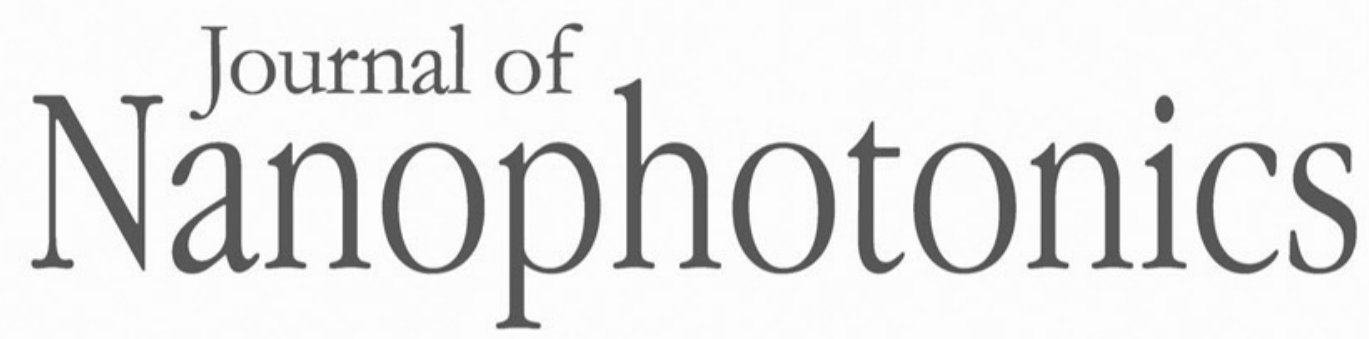

\title{
Cavity-coupled conical cross-section gold nanohole array fiber tip localized surface plasmon resonance sensor
}

\author{
Hong Guo \\ Junpeng Guo
}




\title{
Cavity-coupled conical cross-section gold nanohole array fiber tip localized surface plasmon resonance sensor
}

\author{
Hong Guo and Junpeng Guo* \\ University of Alabama in Huntsville, Department of Electrical and Computer Engineering, \\ Huntsville, Alabama, United States
}

\begin{abstract}
A conical cross-section gold nanoholes array is fabricated on a multi-mode optical fiber tip with an electron-beam lithography process. Underneath the conical gold nanoholes, nanocavities are etched into the substrate fiber glass to increase the light-matter interaction volume. Localized surface plasmon resonance of the fabricated fiber optical device is characterized by measuring the optical transmittance through the fiber tip in the visible and near infrared spectral range. The fiber optical device is used as an index of refraction sensor to detect isopropyl alcohol, ethanol, and methanol. A largely enhanced sensitivity of $652 \mathrm{~nm}$ per refractive index unit due to the conical cross-section gold nanoholes and the nanocavities in the fiber glass substrate is observed. Additionally, the response and recovery times of the fiber-tip plasmon sensor are measured for the first time. (c) The Authors. Published by SPIE under a Creative Commons Attribution 4.0 Unported License. Distribution or reproduction of this work in whole or in part requires full attribution of the original publication, including its DOI. [DOI: 10.1117/1.JNP.14.026006]
\end{abstract}

Keywords: fiber optical sensor; lab-on-fiber; nanophotonics; plasmonics.

Paper 20007 received Jan. 20, 2020; accepted for publication Apr. 1, 2020; published online Apr. 21, 2020.

\section{Introduction}

Localized surface plasmon resonance (LSPR) sensors made of metal nanostructures are a type of "label-free" biochemical sensors, which potentially are useful for many applications such as biochemical sensing, ${ }^{1-5}$ medical diagnostics, ${ }^{6}$ food safety inspection, and environmental monitoring. ${ }^{7-9}$ LSPR label-free sensors utilize the shift of LSPR wavelength caused by surface binding of chemicals as the signal transduction mechanism. Previously, most reported LSPR sensors were made on wafer substrates. Recently, there is increasing interest of integrating LSPR sensors onto optical fibers to create the "lab-on-fiber" sensor platform. ${ }^{10-23}$ "Lab-on-fiber" sensor platform eliminates the need for free space optical alignment, which has many advantages over the traditional "lab-on-chip" sensor platform. Previous works on "lab-on-fiber" technologies have been comprehensively reviewed recently in Refs. 24-26. Among the "lab-on-fiber" sensors, fiber-tip surface plasmon resonance sensors utilize LSPR of metal nanostructures on fiber tips for sensing applications. Various fabrication techniques including focused ion-beam etching, e-beam lithography, and nanopattern transfer have been reported to fabricate LSPR nanostructures on fiber tips. ${ }^{3,13,19,20,22,23}$ In this paper, we report a new fiber-tip nanostructure device for sensing applications. Our new fiber-tip sensor consists of an array of conical cross-section gold nanoholes on a multimode fiber tip and an array of nanocavities underneath in the fiber glass substrate. The fabricated fiber-tip LSPR device was characterized by measuring the optical transmission while different liquid chemicals were applied on the surface. It has been demonstrated that the new fiber-tip LSPR sensor has a higher sensitivity than most previously reported fiber-tip LSPR sensors. ${ }^{3,19,21-23}$ The enhanced sensitivity is verified by rigorous finitedifferent time-domain (FDTD) numerical simulations. It is explained that both the conical shape of gold nanoholes and nanocavities in the fiber substrate contribute to the enhanced sensitivity. Additionally, the response time and recovery time of the fiber-tip plasmonic sensor are measured for the first time per our knowledge.

*Address all correspondence to Junpeng Guo, E-mail: guoj@uah.edu 


\section{Fiber-Tip Nanohole Array Device Fabrication}

The fiber-tip nanohole device fabrication is illustrated in Fig. 1. First, a multimode optical fiber (Corning Infinicor SX+50/125) with a core diameter of $50 \mu \mathrm{m}$ and cladding diameter of $125 \mu \mathrm{m}$ was cleaved to have a flat facet. This type of optical fiber was chosen because of its excellent optical transmission in the visible and short-wave IR spectral range. On the well-cleaved fiber tip, a 1.5-nm thick titanium layer was first deposited as an adhesion layer with a Denton sputter machine. The fiber tip was then dipped into a diluted ZEP-520A e-beam resist (1:1 ratio of ZEP520A to anisole solution) and followed by multiple mechanical vibrations for forming a uniform thin layer of e-beam resist on the fiber facet. The e-beam resist-coated fiber tip was baked in a $120^{\circ} \mathrm{C}$ oven for $30 \mathrm{~min}$. The thickness of the e-beam resist layer is about $100 \mathrm{~nm} .{ }^{3} \mathrm{Using}$ e-beam lithography direct writing, an array of nanoholes was patterned on the e-beam resist layer on the fiber facet. The device was dipped in an e-beam resist developer (ZED-N50) for $1 \mathrm{~min}$ and then was rinsed with deionized water. After that the device was postbaked in the $120^{\circ} \mathrm{C}$ oven for another $30 \mathrm{~min}$. To transfer the nanohole pattern from the e-beam resist layer to the fiber tip, reactive-ion etching (RIE) (Plasma Thermal 790) was used to etch down the developed nanoholes into the fiber glass. The pressure for the RIE etching was 40 mTorr. The radio frequency power was $270 \mathrm{~W}$. The oxygen gas flow rate was 4 standard cubic centimeters per minute (sccm) and the carbon tetrafluoride gas flow rate was $40 \mathrm{sccm}$. After 5-min RIE etching, nanocavities with 200-nm depth were created in the fiber glass. Since the ratio of the etching rates for glass and e-beam resist is 3:1, a layer of e-beam resist between 30 to $40 \mathrm{~nm}$ thickness, was left on the fiber tip after the RIE etching. Then a 60 -nm thick gold film was deposited on the fiber tip device surface in a Denton sputter machine with a sputtering angle of about 50 deg with respect to the surface normal on a rotational holding stage. The sputtered gold thin film was deposited on the sidewall and top surface of the photoresist, protecting the photoresist from chemical etching during the chemical sensing. The gold deposition process is illustrated in Fig. 1(c). After depositing a 60 -nm gold film, transmittance from the fiber device was measured with a broadband light source and an optical spectrometer. After the measurement, an additional 30-nm gold film was

(a)

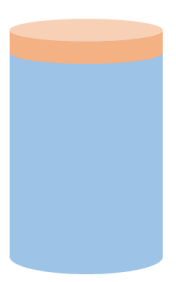

(b)

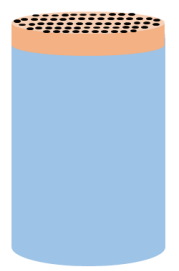

Rotational oblique angle deposition

(d)

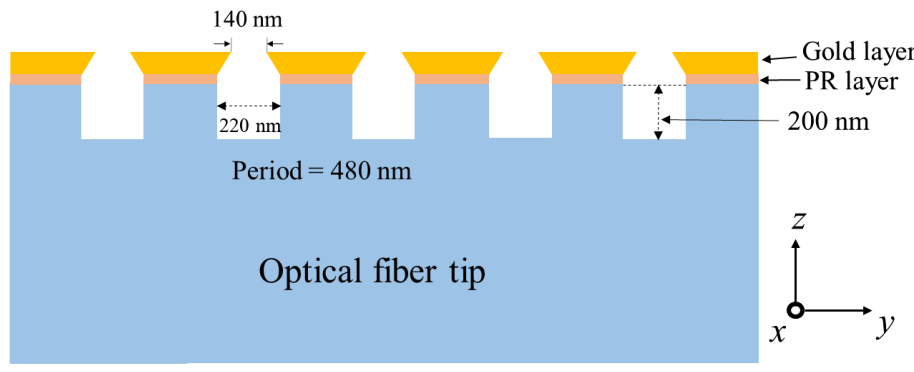

Fig. 1 Fiber-tip nanohole device fabrication process: (a) vibrational coating of an e-beam resist layer on the flat facet of a multimode optical fiber, (b) e-beam lithography patterning of nanoholes and subsequent development and RIE into the fiber glass substrate, (c) rotational oblique angle deposition of gold film on nanohole surface, and (d) the cross section of the conical shape nanoholes. 

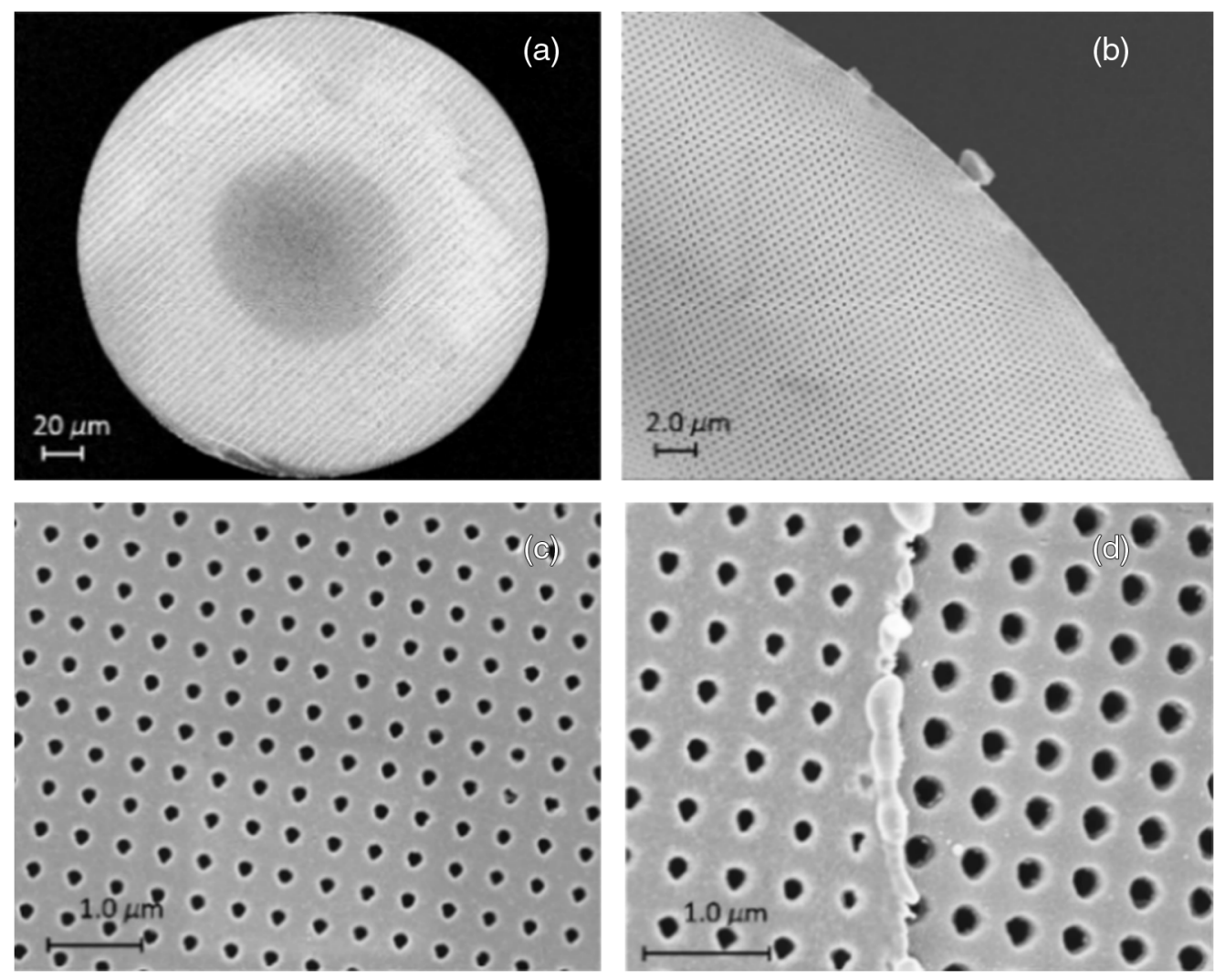

Fig. 2 (a) SEM image of nanohole array patterned in an e-beam resist layer on an optical fiber facet. (b) SEM image of the fabricated device with a 90-nm thick gold film deposited near the fiber edge area. (c) SEM image of the fabricated device with a 90-nm thick gold film deposited in the fiber core area. (d) SEM image of a selected area on the edge of the fiber tip where the right half of the picture shows the metal film was peeled off.

deposited on the fiber tip device surface with the same process illustrated in Fig. 1(c). Figure 1(d) shows the schematic cross section of the conical nanohole array structure.

Scanning electron microscope (SEM) images of fabricated nanoholes on a fiber tip are shown in Fig. 2. Figure 2(a) shows the SEM image of the nanohole array pattern of the e-beam resist layer. Figures 2(b) and 2(c) show the SEM images of the fabricated device with a 90-nm gold film on the edge and the core area of the fiber-tip, respectively. The period of the nanohole is $480 \mathrm{~nm}$ in both dimensions. The depth of the nanocavities etched into the fiber glass is $200 \mathrm{~nm}$. The diameter of the etched nanocavities is about $220 \mathrm{~nm}$. After deposition of the 90 -nm gold film, the diameter of the aperture of the conical cross-section holes on the top is $140 \mathrm{~nm}$. To validate the fabrication process, the gold film was peeled off near the edge of the fiber tip for comparison. In Fig. 2(d), it can be seen that the nanoholes on the top of the metal film (left) and nanoholes in the bottom of the gold film on the e-beam resist layer (right) have different sizes. Nanoapertures on the left have a smaller size than the nanoapertures on the right. The size difference was due to the rotational oblique angle deposition of the gold film.

\section{Measurement Results and Discussion}

After a 60-nm gold layer was deposited on the fiber facet, transmittance through the fiber-tip device was measured using a broadband halogen light source and an optical spectrometer (StellarNet, Inc.). The measured transmittance is shown as the blue line curve in Fig. 3(a). After the measurement, an additional 30-nm gold film was deposited using the same technique and the optical transmittance was measured again. Transmittance corresponding to a 90 -nm gold film is also shown as the black line curve in Fig. 3(a). Two surface plasmon resonance modes 

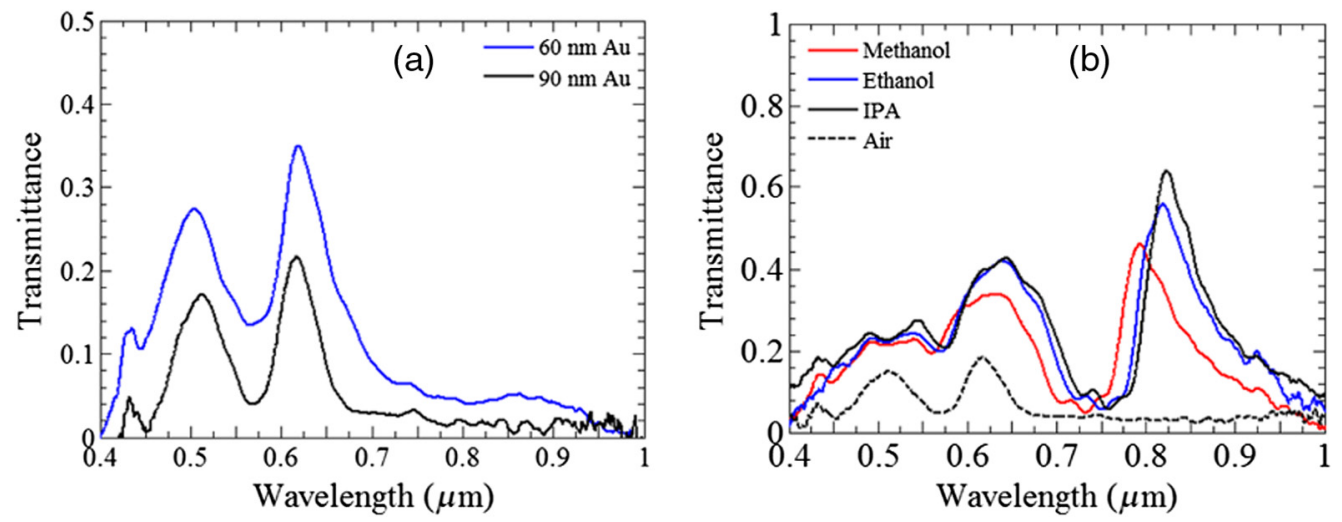

Fig. 3 (a) Measured optical transmission spectra of fabricated conical cross-section fiber-tip nanohole devices with 60- and 90-nm thick gold films in the air $(n=1.0)$. (b) Measured optical transmission spectra of the $90-\mathrm{nm}$ gold layer fiber-tip device when three different chemicals are applied on the fiber sensor tip.

were observed in the transmittance curves. One is at the 510-nm wavelength, which is due to the intrinsic material resonance of gold film. Another occurs at the wavelength of $614 \mathrm{~nm}$ for the 60-nm gold film and $616.5 \mathrm{~nm}$ for the $90-\mathrm{nm}$ thick gold film. It can be seen that the spectral linewidth of this resonance mode becomes narrower as the gold layer changes from 60 to $90 \mathrm{~nm}$ and the peak transmittance decreases as the gold layer becomes thicker. The transmission peak wavelength changes very little with gold film thickness. The dependence of the resonance wavelength on gold layer thickness follows the same trend of localized plasmon resonance in cylindrical gold nanohole arrays. ${ }^{27}$

The fabricated fiber device with a 90-nm gold film was used as a refractive index sensor. In the optical setup, the fiber tip points down from the top so that an optical fiber collimator placed below can collect the transmission light through fiber-tip nanoholes. A microscope glass slide is put between the fiber tip and the collimator so it can hold chemical liquids. The fiber tip can move up and down using a translation stage so that the fiber tip can dip into liquid on the glass slide. The transmitted light collected was sent to an optical spectrometer. Methanol, ethanol, and isopropyl alcohol (IPA) liquids with refractive indices of 1.330, 1.366, and 1.377, respectively, were used for the experiments. During the measurements, each chemical was dropped on the glass slide and the transmission spectrum was measured. Figure 3(b) shows the measured transmittance spectra after applying different liquid chemicals (the solid line curves) to the fiber-tip device. For comparison, the dash line curve in Fig. 3(b) is the transmittance spectrum of the fiber device in air. It can be seen that after a liquid chemical was applied on the fiber tip, a large transmission peak appears in the near-infrared region. This large transmission peak corresponds to the fundamental mode of the LSPR of the gold nanohole array device. The small transmission peak on the left corresponds to the second-order surface plasmon resonance mode. As the chemical was changed, the peak transmission wavelength shifted accordingly. The peak transmission wavelength is $789.0 \mathrm{~nm}$ for methanol of $n=1.330,812.5 \mathrm{~nm}$ for ethanol of $n=1.366$, and $819.5 \mathrm{~nm}$ for IPA of $n=1.377$. It can be seen that the peak transmission wavelength of the fundamental mode resonance is very sensitive to the change of chemicals with different indices of refraction. The peak transmission wavelength shifted from 789 to $812.5 \mathrm{~nm}$ as the methanol was replaced with ethanol. The refractive index difference between ethanol and methanol is 0.036. Therefore, the sensitivity is $653 \mathrm{~nm}$ per refractive index unit (RIU). When the ethanol was replaced with IPA, the peak transmission wavelength shifted from 812.5 to $819.5 \mathrm{~nm}$. The sensitivity corresponding to this resonance wavelength shift is $636 \mathrm{~nm} / \mathrm{RIU}$. This sensitivity is higher than all previously reported sensitivities of cylindrical gold nanohole array surface plasmon resonance sensors in the same spectral range. ${ }^{21-23,28-31}$ The measured sensitivities are summarized in Table 1.

The response and recovery times of the fiber-tip plasmon resonance sensor are characterized in the first time with a musical metronome. In the measurement, the temple of the metronome was set to 3 beats per second. The transmittance spectrum and metronome beats were recorded 
Table 1 Sensitivities and response and recovery times of the LSPR optical fiber tip sensor.

\begin{tabular}{lccccc}
\hline \hline Chemical & $N$ & $\lambda(\mathrm{nm})$ & Sensitivity $(\mathrm{nm} / \mathrm{RIU})$ & Response time $(\mathrm{s})$ & Recovery time (s) \\
\hline Methanol & 1.330 & 789.0 & - & 0.84 & 2.7 \\
Ethanol & 1.366 & 812.5 & 652 & 0.96 & 4.1 \\
IPA & 1.377 & 819.5 & 636 & 0.36 & 3.7 \\
\hline \hline
\end{tabular}

with a rate of $30 \mathrm{~ms}$ per frame. When the measurement started, we started to count the metronome beats and counted to the 9 th metronome beat (last $3 \mathrm{~s}$ ). At the 10th beat, the fiber-tip device was dipped into the liquid. After the transmission spectrum was stabilized, we counted another 9 beats and removed the fiber tip from the chemical liquid at exactly the 10th beat. The response time is the time duration starting from when the fiber tip contacted the chemical liquid to the time when the transmission spectrum became stabilized. The recovery time is the time duration when the fiber tip leaves the liquid to the time when the transmission spectrum recovered to the transmission spectrum in air. By counting the number of the beats of the metronome, the response times for methanol, ethanol, and IPA are 0.84, 0.96, and $0.36 \mathrm{~s}$, respectively. The recovery times for methanol, ethanol, and IPA to completely evaporate were $2.7,4.1$, and $3.7 \mathrm{~s}$, respectively. The measured response and recovery times are also summarized in Table 1 . The response and recovery times vary with chemicals, but are in the order of seconds. The fast recovery is due to the local heating effect of the surface plasmon resonance. ${ }^{32,33}$

To understand the effects of the conical shape of nanoholes and nanocavities on the LSPR, FDTD simulations were carried out to calculate the transmittance spectrum and the electric field distribution using a software code (Lumerical Solutions, Inc.). In the simulations, the boundaries were set as periodic boundaries in $x$ and $y$ directions. A perfect matched layer (PML) boundary was set in the $z$ direction. In the simulated structure, the gold layer thickness is set at $90 \mathrm{~nm}$, the period of the square lattice nanohole array is $480 \mathrm{~nm}$, and optical constants of gold film and silicon dioxide are from the Palik's Handbook of Optical Constants. ${ }^{34}$ To obtain electric field distributions, a two-dimensional (2-D) field monitor is placed on the cross section of a nanohole in the $y-z$ plane. A conical-shape nanohole array with a top radius of $70 \mathrm{~nm}$ and a bottom radius of $110 \mathrm{~nm}$ sits on a glass substrate with a 200-nm deep cavity below each conical cross-section gold nanohole. The transmittance spectra for different top aperture sizes are plotted and shown in Fig. 4(a). With the bottom aperture diameter fixed at $220 \mathrm{~nm}$, optical transmittance was calculated for different top aperture diameters from 0 to $220 \mathrm{~nm}$. The index of refraction of the medium in the hole and the surrounding is 1.40 in simulations. It can be seen that as the top aperture size decreases, the spectral width of the fundamental mode decreases and the peak
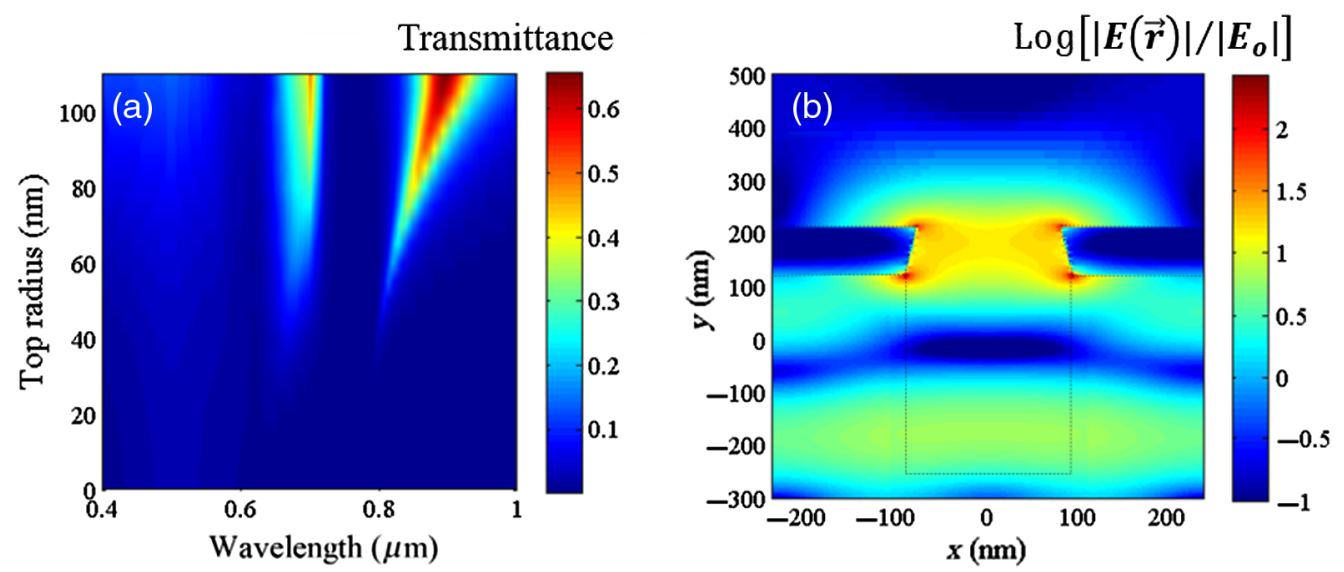

Fig. 4 (a) Calculated optical transmission spectra of conical cross-section nanoholes with different top aperture radii from 0 to $110 \mathrm{~nm}$. (b) Simulated electric field enhancement profile at the peak transmission wavelength of $850 \mathrm{~nm}$ plotted in logarithmic scale. 
transmittance also decreases as shown in Fig. 4(a). The fundamental plasmon resonance mode has a blue shift and the second-order resonance mode stays at about the same wavelength.

We also calculated the electric field distribution on the conical cross section of a nanohole at the fundamental mode resonance wavelength of $850 \mathrm{~nm}$. In the structure, the top diameter of the nanohole aperture is $140 \mathrm{~nm}$ and the bottom diameter of the nanohole aperture is $220 \mathrm{~nm}$. Figure 4(b) shows the calculated electric field enhancement in logarithmic scale. The electric field enhancement is the ratio of the calculated electric field amplitude over the incident plane wave electric field amplitude. The black dash line shows a nanocavity below a conical shape nanohole in the gold film. It can be seen that the electric field inside the conical cross-section gold nanohole is significantly enhanced at the fundamental LSPR wavelength. A maximal electric field enhancement factor of two orders of magnitude is observed at the peak transmission wavelength of $850 \mathrm{~nm}$.

Both the conical shape nanoholes and the nanocavities contribute to the large sensitivity. To explain the enhanced sensitivity, FDTD simulations were carried out to calculate the transmission spectra of devices with cylindrical shape and conical shape nanoholes, and with and without nanocavities. Transmission spectra from a conical shape nanohole array and from a cylindrical shape nanohole array without cavities were calculated for different surrounding media with refractive indices $n=1.0,1.36,1.50$, respectively. The cross sections of a conical nanohole and a cylindrical nanohole are shown in the inset of Fig. 5(a). The nanohole aperture radius at the bottom is $110 \mathrm{~nm}$ and the nanohole aperture radius at the top is $70 \mathrm{~nm}$. For the cylindrical nanohole array, the nanohole radius is $110 \mathrm{~nm}$. The calculated transmission spectra are plotted and shown in Fig. 5(a). For the conical cross-section nanohole array, the transmittance spectra are the solid line curves with peak transmission wavelengths at 806,832 , and $849 \mathrm{~nm}$ for $n=1.0,1.36,1.50$, respectively. The shift of the peak transmission wavelength shift is $43 \mathrm{~nm}$ from air ( $n=1.0$ ) to $n=1.50$. From $n=1.36$ to $n=1.50$, the peak transmission wavelength shift is $17 \mathrm{~nm}$. For the cylindrical cross-section nanohole array, the peak transmission wavelengths are 806, 826, and $840 \mathrm{~nm}$ for $n=1.0,1.36,1.50$, respectively. The peak transmission wavelength shift is $34 \mathrm{~nm}$ from air $(n=1.0)$ to $n=1.50$, and $14 \mathrm{~nm}$ from $n=1.36$ to $n=1.50$. It is clearly seen that the conical cross-section nanohole array gives a larger peak transmission wavelength shift than that of the cylindrical cross-section nanohole array for the same amount of change of the index of refraction. Therefore, the conical cross-section nanohole array gives higher sensitivity for the index of refraction sensing. In addition, from Fig. 5(a), it can be seen that the conical nanohole array has a higher peak transmittance than that of the cylindrical nanohole array for the high index of refraction surrounding medium. A similar finding was reported earlier in reference. ${ }^{35}$

Transmission spectra of a conical nanohole array with and without nanocavities were calculated for surrounding media of different indices of refraction. The depth of the nanocavities is
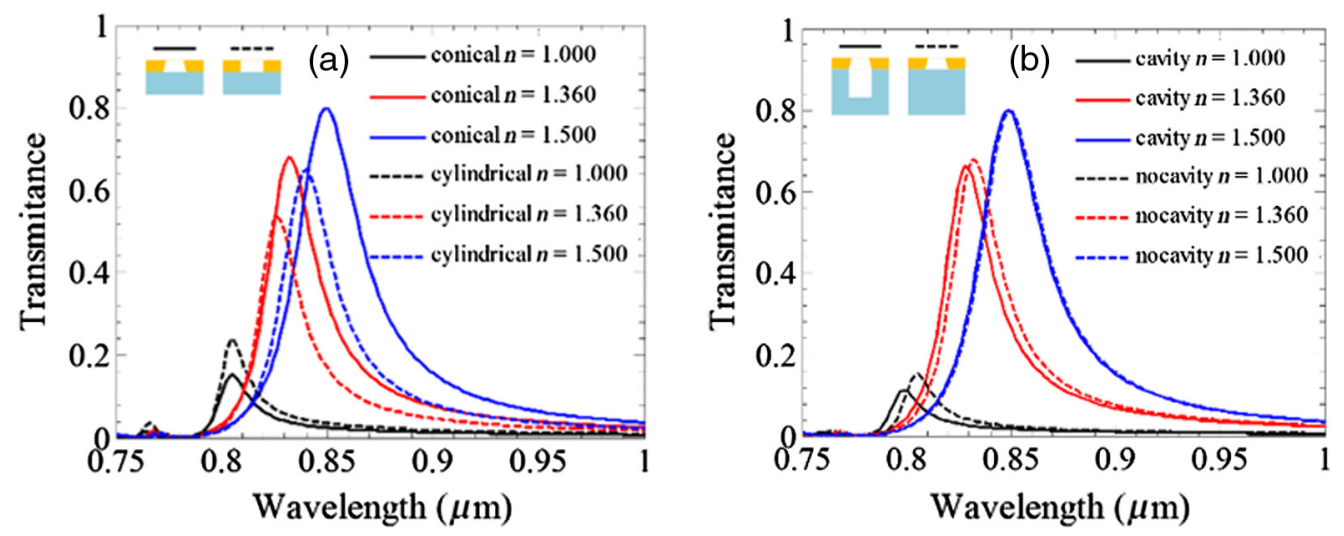

Fig. 5 (a) Calculated transmittance spectra of conical nanohole arrays (solid lines) and cylindrical nanohole arrays (dashed lines) for different indices of refraction of surrounding media. (b) Calculated transmittance spectra of conical nanohole arrays with nanocavities (solid lines) and without nanocavities (dashed lines). 
$200 \mathrm{~nm}$ in the fiber glass. The cross section of gold nanoholes with cavities below is shown in the inset of Fig. 5(b). Calculated transmittance curves are shown in Fig. 5(b). The solid line curves are the transmission spectra of the device with nanocavities, and the dashed line curves are the transmission spectra of the device without cavities for three different surrounding media with indices of refraction $n=1.00,1.36$, and 1.50 , respectively. With nanocavities, the peak transmission wavelengths are 799, 828, and $849 \mathrm{~nm}$, respectively. From $n=1.0$ to $n=1.50$, the device with nanocavities has a 50-nm wavelength shift. From $n=1.36$ to $n=1.50$, the device with nanocavities has a $21-\mathrm{nm}$ wavelength shift. Without cavities, the peak transmission wavelengths are $806,832,849 \mathrm{~nm}$ for $n=1.00,1.36,1.50$, respectively. Without cavities, the device has a 43-nm wavelength shift from $n=1.00$ to $n=1.50$. From $n=1.36$ to $n=1.50$, the device without nanocavities has a shift of a $17-\mathrm{nm}$ wavelength. Comparing the device without nanocavities with the device with nanocavities, the device with nanocavities has a smaller resonance wavelength for low indices of refraction material filling such as $n=1.00$ and $n=1.36$, therefore, it has a larger resonance wavelength shift for increasing the index of refraction of the surrounding medium. The cavities in the substrate cause the blue shift of the resonance wavelength for a lower index of refraction material filling in the cavities. Therefore, nanocavities increase the sensitivity of the sensor. The nanocavities increase the volume of light-matter interactions and can also enhance signals of other types of measurements such as surface enhanced Raman scattering spectroscopy.

\section{Conclusion}

In this work, we fabricated a conical cross-section gold nanohole array device on a flat optical fiber tip. Underneath the conical shape nanoholes, nanocavities were etched into the fiber glass substrate to increase the volume of light-matter interactions. LSPR in the fiber-tip device was characterized by measuring the transmittance spectra with different liquid chemicals applied. Enhanced sensitivity of the fiber-tip LSPR chemical sensor has been demonstrated. The enhanced sensitivity was verified by rigorous FDTD numerical simulations. It has been shown that both conical shape and the nanocavities below contribute to the enhanced sensitivity. Additionally, response time and recovery time of the fiber optical sensor were measured for the first time. The new "lab-on-fiber" LSPR sensor can be potentially used for many chemical and biological sensing applications.

\section{Acknowledgements}

H. Guo acknowledges support from the SPIE Optics and Photonics Education Scholarship Program. J. Guo acknowledges support of the Individual Investigator Distinguished Researcher Award of the University of Alabama in Huntsville. The authors declare no conflicts of interest in the manuscript.

\section{References}

1. K. A. Willets and R. P. Van Duyne, "Localized surface plasmon resonance spectroscopy and sensing," Annu. Rev. Phys. Chem. 58, 267-297 (2007).

2. J. V. Samsonova et al., "Biacore biosensor immunoassay for 4-nonplyphenols assay optimization and applicability for shell fish analysis," Chemosphere 57, 975 (2004).

3. Y. Lin et al., "E-beam patterned gold nanodot arrays on optical fiber tips for localized surface plasmon resonance biochemical sensing," Sensors 10(10), 9397-9406 (2010).

4. H. Guo and J. Guo, "Hybrid plasmon photonic crystal resonance grating for integrated spectrometer biosensor," Opt. Lett. 40(2), 249-252 (2015).

5. J. Guo, Z. Li, and H. Guo, "Near perfect light trapping in a $2 \mathrm{D}$ gold nanotrench grating at oblique angles of incidence and its application for sensing," Opt. Express 24(15), 17259-17271 (2016).

6. J. F. Masson, "Surface plasmon resonance clinical biosensors for medical diagnostics," ACS Sens. 2(1), 16-30 (2017) 
7. M. Li et al., "Nanostructured sensors for detection of heavy metals: a review," ACS Sustain. Chem. Eng. 1(7), 713-723 (2013).

8. E. S. Forzani et al., "Detection of heavy metal ions in drinking water using a high-resolution differential surface plasmon resonance sensor," Environ. Sci. Technol. 39(5), 1257-1262 (2005).

9. D. R. Shankaran, K. V. Gobi, and Norio Miura, "Recent advancements in surface plasmon resonance immunosensors for detection of small molecules of biomedical, food and environmental interest," Sens. Actuators B 121(1), 158-177 (2007).

10. K. Sharma, R. Jha, and B. D. Gupta, "Fiber-optic sensors based on surface plasmon resonance: a comprehensive review," IEEE Sens. J. 7(8), 1118-1129 (2007).

11. A. Leung, P. M. Shankar, and R. Mutharasan, "A review of fiber-optic biosensors," Sens. Actuators B 125(2),688-703 (2007).

12. S. Wolfbeis, "Fiber-optic chemical sensors and biosensors," Anal. Chem. 80, 4269-4283 (2008).

13. G. Kostovski, P. R. Stoddart, and A. Mitchell, "The optical fiber tip: an inherently lightcoupled microscopic platform for micro and nanotechnologies," Adv. Mater. 26, 3798-3820 (2014).

14. C. Caucheteur, T. Guo, and J. Albert, "Review of plasmonic fiber optic biochemical sensors: improving the limit of detection," Anal. Bioanal. Chem. 407, 3883-3897 (2015).

15. S. K. Srivastava and B. D. Gupta, "A multitapered fiber-optic SPR sensor with enhanced sensitivity," IEEE Photonics Technol. Lett. 23(13), 923 (2011).

16. V. V. R. Sai, K. Tapanendu, and M. Soumyo, "Novel U-bent fiber optic probe for localized surface plasmon resonance based biosensor," Biosens. Bioelectron. 24(9), 2804-2809 (2009).

17. M. Tian et al., "All-solid D-shaped photonic fiber sensor based on surface plasmon resonance," Opt. Commun. 285(6), 1550-1554 (2011).

18. H. Y. Lin et al., "Side-polished multimode fiber biosensor based on surface plasmon resonance with halogen light," Appl. Opt. 46(5), 800-806 (2007).

19. M. Consales et al., "Lab-on-fiber technology: toward multi-functional optical nanoprobes," ACS Nano 6(4), 3163-3170 (2012).

20. L. Tian et al., "Transmission properties of nanoscale aperture arrays in metallic masks on optical fibers," J. Appl. Phys. 101, 014303 (2007).

21. P. Jia et al., "Quasiperiodic nanohole arrays on optical fibers as plasmonic sensors: fabrication and sensitivity determination," ACS Sens. 1(8), 1078-1083 (2016).

22. P. Jia and J. Yang, "Integration of large-area metallic nano-hole arrays with multimode optical fibers for surface plasmon resonance sensing," Appl. Phys. Lett. 102(24), 243107 (2013).

23. A. Dhawan et al., "Focused ion beam fabrication of metallic nanostructures on end faces of optical fibers for chemical sensing applications," J. Vac. Sci. Technol. B 26, 2168 (2008).

24. M. Consales, M. Pisco, and A. Cusano, "Lab-on-fiber technology: a new avenue for optical nanosensors," Photonic Sens. 2(4), 289-314 (2012).

25. A. Ricciardi et al., "Lab-on-fiber technology: a new vision for chemical and biological sensing," Analyst 140(24), 8068-8079 (2015).

26. P. Vaiano et al., "Lab on fiber technology for biological sensing applications," Lasers Photonics Rev. 10(6), 922-961 (2016).

27. C. Genet and T. W. Ebbesen, "Light in tiny holes," Nature 445(7123), 39-46 (2007).

28. G. Brolo et al., "Surface plasmon sensor based on the enhanced light transmission through arrays of nanoholes in gold films," Langmuir 20(12), 4813-4815 (2004).

29. D. Leebeeck et al., "On-chip surface-based detection with nano-hole arrays," Anal. Chem. 79(11), 4094-4100 (2007).

30. A. Yanik et al., "Integrated nanoplasmonic-nanofluidic biosensors with targeted delivery of analytes," Appl. Phys. Lett. 96, 021101 (2010).

31. J. P. Monteiro et al., "Effect of periodicity on the performance of surface plasmon resonance sensors based on subwavelength nanohole arrays," Sens. Actuators B: Chem. 178, 366-370 (2013).

32. G. Baffou, R. Quidant, and C. Girard, "Heat generation in plasmonic nanostructures: influence of morphology," Appl. Phys. Lett. 94(15), 153109 (2009). 
33. Z. J. Coppens et al., "Probing and controlling photothermal heat generation in plasmonic nanostructures," Nano Lett. 13(3), 1023-1028 (2013).

34. E. D. Palik, Handbook of Optical Constants of Solids, Academic Press, San Diego, California (1997).

35. M. Diwekar, S. Blair, and M. Davis, "Increased light gathering capacity of sub-wavelength conical metallic apertures," J. Nanophotonics 4(1), 043504 (2010).

Hong Guo received his BS degree in optics from Sichuan University in 2008 and his PhD in electrical engineering from the University of Alabama in Huntsville in 2018. He is a recipient of the 2017 Optics and Photonics Education Scholarship Award from SPIE. Currently, he is a senior process engineer at Enablence Technology, Inc., Fremont, California, USA. His research interests include nanophotonics, plasmonics, silicon photonics, and planar lightwave circuits integrated photonics research and development.

Junpeng Guo received his $\mathrm{PhD}$ in electrical engineering from the University of Illinois at Urbana-Champaign. Since 2005, he has been a professor of electrical engineering and optics at the University of Alabama in Huntsville. He is a fellow of SPIE and currently serves as an associate editor of the Journal of Nanophotonics and also an associate editor of Photonics Research journal. 